\title{
Shifting priorities in the aftermath of a $\mathrm{Q}$ fever epidemic in 2007 to 2009 in the Netherlands: from acute to chronic infection
}

W van der Hoek (Wim.van.der.hoek@rivm.nl)1, P M Schneeberger ${ }^{2}$, T Oomen ${ }^{1}$, M C Wegdam-Blans ${ }^{3}$, F Dijkstra', D W Notermans ${ }^{1}$, H A Bijlmer ${ }^{1}$, K Groeneveld ${ }^{4}$, C J Wijkmans ${ }^{5}$, A Rietveld ${ }^{5}$, L M Kampschreur ${ }^{6}$, Y van Duynhoven ${ }^{1}$

1. National Institute for Public Health and the Environment (RIVM), Centre for Infectious Disease Control, Bilthoven, the Netherlands

2. Jeroen Bosch Hospital, 's-Hertogenbosch, the Netherlands

3. Laboratory for Pathology and Medical Microbiology (PAMM), Veldhoven, the Netherlands

4. Health Council of the Netherlands, The Hague, the Netherlands

5. Municipal Health Service 'Hart voor Brabant', 's-Hertogenbosch, the Netherlands

6. University Medical Centre Utrecht, Department of Internal Medicine and Infectious Diseases, Utrecht, the Netherlands

Citation style for this article:

van der Hoek W, Schneeberger PM, Oomen T, Wegdam-Blans MC, Dijkstra F, Notermans DW, Bijlmer HA, Groeneveld K, Wijkmans CJ, Rietveld A, Kampschreur LM, van Duynhoven Y. Shifting priorities in the aftermath of a Q fever epidemic in 2007 to 2009 in the Netherlands: from acute to chronic infection.

Euro Surveill. 2012;17(3):pii=20059. Available online: http://www.eurosurveillance.org/ViewArticle.aspx?Articleld=20059

Article published on 19 January 2012

From 2007 to 2009, the Netherlands faced large seasonal outbreaks of $Q$ fever, in which infected dairy goat farms were identified as the primary sources. Veterinary measures including vaccination of goats and sheep and culling of pregnant animals on infected farms seem to have brought the $Q$ fever problem under control. However, the epidemic is expected to result in more cases of chronic $\mathrm{Q}$ fever among risk groups in the coming years. In the most affected area, in the south of the country, more than $12 \%$ of the population now have antibodies against Coxiella burnetii. Questions remain about the follow-up of acute $Q$ fever patients, screening of groups at risk for chronic $Q$ fever, screening of donors of blood and tissue, and human vaccination. There is a considerable ongoing research effort as well as enhanced veterinary and human surveillance.

\section{Introduction}

Acute $Q$ fever was made mandatorily notifiable in the Netherlands in 1975, but was rarely reported from 1975 to 2006 (with between one and 32 notifications per year). In 2005, Q fever was diagnosed on two dairy goat farms with unusually high numbers of abortions and two years later, in 2007, it emerged in the human population in the south of the Netherlands. This was the start of an exceptionally large epidemic that showed a marked seasonality and expanded both geographically and in size in 2008 and 2009. From 2007 to 2009 , more than 3,500 human cases were notified. The observation that human cases mainly occurred in the same area as dairy goat farms with $\mathrm{Q}$ fever-induced abortion waves provided circumstantial evidence that dairy goat farms were the most plausible source of human infection in this epidemic. The patients most affected were men, smokers and aged 40-60 years, while children were rarely affected [1]. Acute Q fever mainly presents as febrile illness, pneumonia or hepatitis, but clinical presentation may vary from one area to another [2]. More than $92 \%$ of notified patients in the Netherlands with onset of illness in 2007 and 2008 had fever, while $62 \%$ presented with pneumonia [1]. Hepatitis was reported in less than $1 \%$ of notified patients but is a common presentation of acute $Q$ fever in some countries such as France [2]. The diagnosis $Q$ fever can only be made after confirmation with a laboratory test. Serological methods can detect antibodies against phase I and phase II antigens of Coxiella burnetii, the causative agent of $\mathrm{Q}$ fever, and thereby distinguish acute from chronic disease. Annual updates on the $Q$ fever epidemic in the Netherlands have been published in this journal [3-5]. We now report on the current situation in the aftermath of the epidemic, focusing on the challenges and remaining questions, especially with respect to chronic Q fever.

\section{Decreasing incidence of acute $Q$ fever, increasing seroprevalence}

The epidemiological situation in the aftermath of the epidemic can be characterised by a decreased incidence of notifications of acute $Q$ fever and an increased prevalence of antibodies to $C$. burnetii in the general population, particularly in the most affected area in the south of the country. The number of notified acute $Q$ fever patients fell from 2,354 in 2009 to 504 in 2010 (Figure). From January to November 2011, 81 patients were notified, which is far fewer than the same period in the epidemic years, despite the exceptionally warm and dry weather conditions in the spring of 2011, which are considered conducive to airborne spread of C. burnetii. 
It is difficult to attribute the decrease in incidence in 2010 and 2011 to any particular control measure because several veterinary interventions were implemented at the same time. In April 2009, vaccination of sheep and goats on dairy farms with more than 50 animals and on farms with public functions in the highincidence area became mandatory and was extended to the entire country in January 2010 [6]. In addition, stringent hygiene measures were implemented, such as safe manure management and hygiene during lambing. In October 2009, mandatory monitoring of bulk tank milk was implemented. In addition, from December 2009 to June 2010, more than 50,000 pregnant goats and sheep were culled on 87 farms in which bulk tank milk was positive for $C$. burnetii.

Increasing immunity and thereby a smaller population at risk among the general population in the highincidence area might also have played a role in the decrease in incidence of acute $Q$ fever. Seroprevalence among the general population of the Netherlands was only $2.4 \%$ during February 2006 to May 2007, before the first outbreak in June 2007 [7]. More recent nationwide figures are not available, but in the high-incidence area, seroprevalence estimates are available for pregnant women in 2007 to 2009 (9.0\%) [8] and for blood donors in 2009 (12.2\%) [9].

\section{Chronic Q fever}

Despite the decreasing incidence of acute $Q$ fever, the $Q$ fever problem is not over: a rising number of chronic $Q$ fever patients are seen. An estimated $2 \%$ of acute $Q$ fever patients develop chronic $Q$ fever months to years after the acute infection [10]. Chronic Q fever mainly presents as endocarditis or vascular infection and carries a high morbidity and mortality. Infected patients with previous cardiac valve pathology, aneurysms or vascular grafts or who are immunocompromised and women who are infected during pregnancy are most at risk of developing chronic $Q$ fever [2]. Diagnosis of chronic $Q$ fever is based on a combination of the following: PCR analysis positive for $C$. burnetii in blood or tissue in the absence of an acute infection, an IgG phase I antibody titre of $\geq 1: 1,024$, presence of clinical risk factors, presence of clinical signs, and radiological imaging results including echocardiography and positron emission tomography-computed tomography (PET-CT) [11]. There is no notification system for chronic $Q$ fever in the Netherlands, but based on personal communications from various Dutch hospitals in September 2011, we estimate that a total of over 250 patients have been diagnosed since the start of the epidemic.

The major challenge in the Netherlands is therefore early detection and treatment of patients who are at risk for chronic $Q$ fever. The following issues are of particular relevance: (i) the follow-up strategy of acute $Q$ fever patients, for the early detection and prompt treatment of chronic Q fever; (ii) the screening of people in risk groups for chronic $Q$ fever; (iii) the protection of people in risk groups through vaccination; and (iv) the possibility of person-to-person transmission through infected blood or tissue.

\section{FIGURE}

Acute Q fever notifications, the Netherlands, 1 January (week 1) 2007-30 November (week 48) 2011

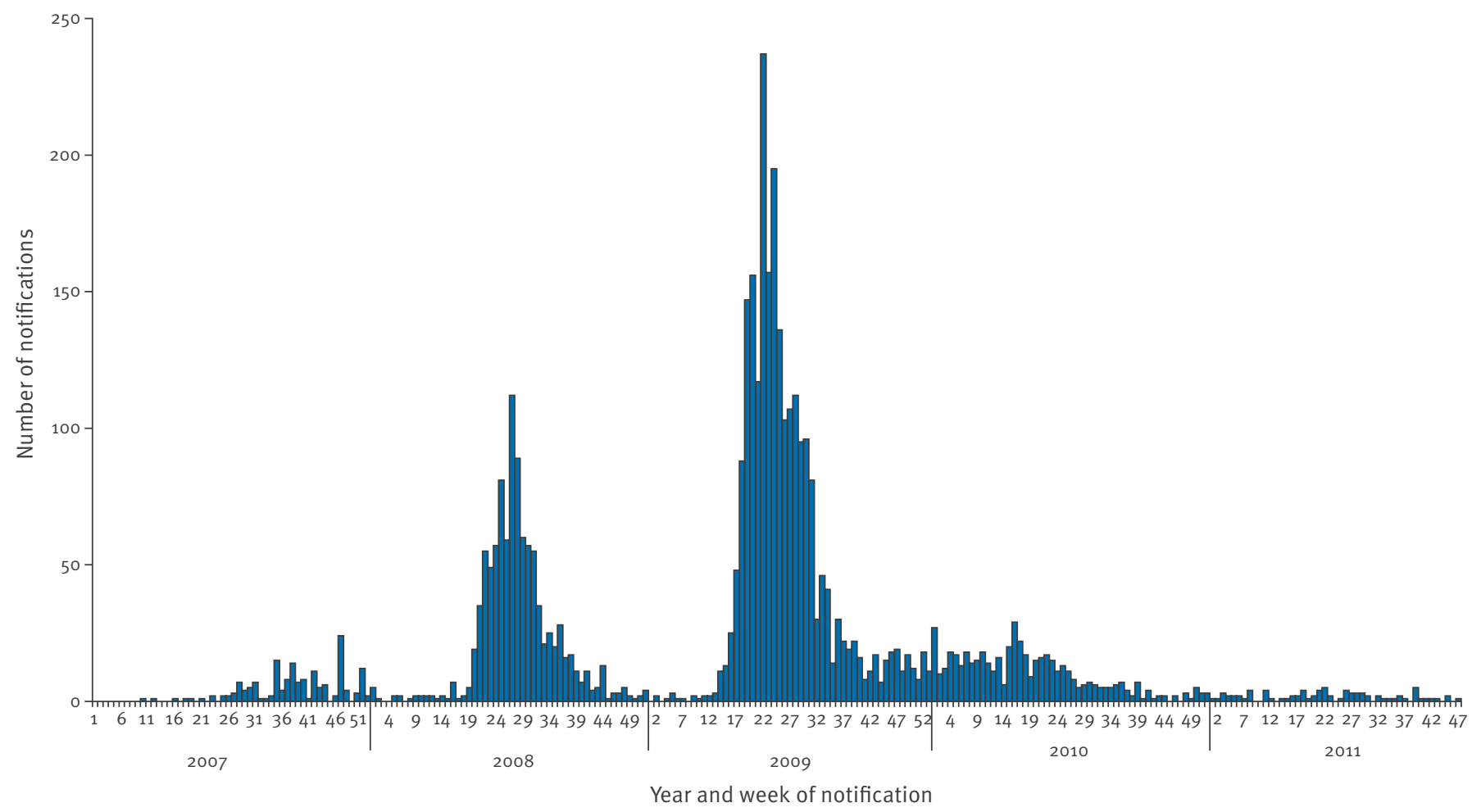


It is expected that the number of patients with chronic infection will increase in the Netherlands the coming years. In order to diagnose and treat chronic $Q$ fever patients in a consistent way, new guidelines are currently being developed for the diagnosis of chronic $Q$ fever in the country.

\section{Follow-up of acute $Q$ fever patients}

In the early stages of the epidemic, the internationally recommended follow-up strategy was followed, consisting of at least three consecutive serological tests in the first year after the diagnosis of acute $Q$ fever and echocardiography for all patients diagnosed with acute Q fever [12]. However, of 134 Dutch Q fever patients from the 2007 and 2008 outbreaks who had undergone screening echocardiography and were followed up for one year after diagnosis of acute infection, none progressed to a chronic infection and echocardiographic screening was discontinued [13]. However, the policy of discontinuing echocardiographic screening has been challenged by Raoult et al., on the basis of data from France that show that clinically silent valvulopathies predispose to chronicity [14]. Considerable uncertainties also exist about the value of serology to identify chronic cases during follow-up. At the regional laboratory of the Jeroen Bosch Hospital (in 's-Hertogenbosch), located at the epicentre of the Dutch outbreaks, the serological profiles of 686 patients diagnosed with acute $Q$ fever in 2007 and 2008 were evaluated at three, six and 12 months after diagnosis [15]. The results differ from data provided by others, as high IgG phase I antibody titres at the three-month follow-up were not predictive for chronic Q fever and IgG phase I antibody titres greater than IgG phase II antibody titres were rarely seen. The study confirmed that a cut-off value of $\geq 1: 1,024$ for IgG phase I titres is suitable for screening in the commercially available immunofluorescence assay used (Focus Diagnostics, United States), at a follow-up between six and 12 months after the acute $Q$ fever episode. For patients with clinical risk factors, however, a more stringent follow-up scheme is required. Wide variation in serological and PCR test results during the follow-up of acute $Q$ fever [15] implies that the diagnosis of chronic $Q$ fever - necessitating long-term antibiotic treatment - must be based on a combination of laboratory results, radiological imaging and clinical grounds. On the basis of the experience gained since 2007, the follow-up strategy is now generally one serological analysis nine months after an episode of acute $\mathrm{Q}$ fever. For patients with specific risk factors, the previous serological follow-up strategy at three, six and 12 months is maintained, with use of PCR if high IgG I titres are obtained.

\section{Screening of risk groups for chronic $Q$ fever} Chronic Q fever has been diagnosed in the Netherlands in patients who had no history of acute $Q$ fever, suggesting that chronic $Q$ fever can develop after asymptomatic infection or symptomatic infection with only mild aspecific symptoms. The incubation period of a chronic infection is largely unknown and may be different in patients with vascular disease compared with those who have valvular disease. Some hospitals in the high-incidence area are now implementing screening programmes for the detection of chronic $Q$ fever in patients with known cardiac valve or vascular pathology. The risk of chronic $Q$ fever in other risk groups, such as pregnant women, is probably too low to warrant a targeted screening strategy.

\section{Human vaccination}

$\mathrm{Q}$ fever can be prevented by a vaccine that is produced and licensed in Australia to protect abattoir workers [16]. For this and other occupational risk groups, such as sheep shearers and farmers of ruminants, the vaccine has proved to be successful and is still in use in Australia [17]. From the notification data, it is clear that occupational exposure did not play an important role in the epidemic in the Netherlands [1]. The prevalence of antibodies against $C$. burnetii in dairy goat farmers and practising veterinarians is greater than $80 \%$, but very few seem to develop clinical disease (unpublished data). Vaccination - a one-off campaign during the epidemic - was therefore primarily considered for persons at risk for chronic Q fever. Implementing vaccination was difficult, however, because the vaccine is not registered in any European country and its effectiveness has only been shown in healthy young adults, not in persons with cardiovascular risk factors or patients with severe underlying disease [18]. Moreover, the logistics are cumbersome: the vaccine can only be given to those who have not previously been in contact with $C$. burnetii, as vaccinating people who have already mounted an immune response against the pathogen may lead to serious adverse reactions such as sterile abscesses and systemic symptoms of inflammation. Therefore, serology and skin testing are mandatory before vaccination. In the absence of a licence, the vaccine can only be administered after the patients' physician has signed a medical awareness statement and the patient has signed an informed consent form. Nevertheless, the Health Council of the Netherlands advised vaccination of people in specific risk groups in the high-incidence area who have an increased risk of developing complications following acute infection [19]. The groups included patients:

- who have had endocarditis

- with prosthetic heart valves

- with important congenital heart anomalies, including those that required grafts

- with structural defects of the aortic or mitral valve

- with known aneurysm of the aorta

- with vascular grafts

- with severe peripheral vascular disease (such as Buerger's disease).

General practitioners selected all patients from these groups from their patient registration systems. In total, 1,781 patients were screened: 394 (22\%) could not be vaccinated because of a positive skin test or the presence of antibodies against C. burnetii. After 
screening, 21 eligible patients declined vaccination or did not attend the vaccination session: eventually 1,366 patients were vaccinated from 28 January to 27 June 2011. There is a routine follow-up of vaccinated individuals for vaccine-related adverse events - the results should be available by the end of 2011 . The vaccination campaign has also been followed by a postvaccination immune-response study in which humoral and cell-mediated immunity will be investigated.

\section{Transmission of C. burnetii by infected blood and tissue}

Although only few cases have been clearly documented, there is a theoretical possibility that $C$. burnetii can be transmitted through blood transfusion, and semen, tissue and organ donation [20]. Active screening was therefore recommended by the European Centre for Disease Prevention and Control (ECDC) in their risk assessment in 2010 [10]. Sanquin Blood Supply Foundation tested blood donated from people living in the area with highest $Q$ fever incidence in the south of the Netherlands for the presence of $C$. burnetii DNA by PCR from 20 May 2009 - in 2009 as part of a research project, then in 2010 as a screening instrument [9]. In 1,004 blood donations, there were three positive PCR results and in one recipient, there was evidence of seroconversion. However, the recipient lived in the high-incidence area and it is therefore possible that the infection was caused by environmental exposure. The screening programme was discontinued on 1 November 2010, when it was clear that the incidence of the disease had fallen dramatically. With the decreasing incidence and the expected increasing numbers of chronic infections in the coming years, the issue of protecting recipients of blood, semen, tissue and organs is shifting towards detecting asymptomatic persons harbouring $C$. burnetii months to years after their acute infection. However, there are important logistic and financial constraints in using PCR on a large scale. Capacity for PCR testing at Sanquin is limited to 100 samples per day, while close to a million blood component transfusions are given annually. Alternatively, donors could be screened for the presence of IgG phase I antibodies against $C$. burnetii. For large-scale screening purposes, an automated ELISA would have to be used, but the performance of ELISAs for IgG phase I antibodies have yet to be evaluated.

In August 2011, the Health Council of the Netherlands advised that a detailed cost-effectiveness analysis of serological testing of blood donors be carried out and, should the incidence of acute $Q$ fever increase again, screening of blood donors be resumed [20]. Concerning tissue donations, no screening is needed for tissues that carry a low risk of transmission such as cornea, coagulants and other treated blood products or tissues collected before 2007. Otherwise, nationwide serological testing is recommended. In certain circumstances, such as organ transplantation or use of stem cells, the responsible physician and patient might decide to use infected material anyway, when a considerable improvement in quality of life or even the saving of life is anticipated. Knowing that the donor's serological status is positive can then make appropriate antibiotic prophylactic treatment of the recipient possible.

\section{Persistent fatigue after acute $Q$ fever}

While relatively few patients who have had acute infection develop chronic $Q$ fever, a much larger group suffers from persistent fatigue and other long-term effects of acute infection. Unlike chronic $Q$ fever, this is not a life-threatening condition, but the fatigue can be debilitating and seriously affect the person's quality of life [21]. In an ongoing study, the effectiveness of antibiotic treatment is being compared with cognitive behavioural therapy for post acute $Q$ fever fatigue.

\section{Outlook}

We expect that the sustained mandatory vaccination of goats and sheep will control transmission of $Q$ fever to humans. The veterinary vaccine seems effective in reducing shedding of $C$. burnetii and in preventing abortion [22]. However, the bacteria are widespread in the environment and in other animal reservoirs, such as wild rats [23]. Enhanced surveillance in animal populations as well as in humans will remain essential. To fill the remaining knowledge gaps, there is an extensive ongoing research agenda, covering fields such as as host-pathogen characteristics, transmission and risk factors, chronic $Q$ fever and treatment of post acute $Q$ fever fatigue.

\section{References}

1. Dijkstra F, van der Hoek W, Wijers N, Schimmer B, Rietveld A, Wijkmans CJ, et al. The 2007-2010 Q fever epidemic in the Netherlands: characteristics of notified acute $Q$ fever patients and the association with dairy goat farming. FEMS Immunol Med Microbiol. 2011. doi: 10.1111/j.1574-695X.2011.00876.x. [Epub ahead of print].

2. Maurin M, Raoult D. Q fever. Clin Microbiol Rev. 1999;12(4):518-53.

3. Schimmer B, Morroy G, Dijkstra F, Schneeberger PM, Weers-Pothoff $G$, Timen A, et al. Large ongoing $Q$ fever outbreak in the south of the Netherlands, 2008. Euro Surveill. 2008;13(31):pii=18939. Available from: http://www. eurosurveillance.org/ViewArticle.aspx?Articleld=18939

4. Schimmer B, Dijkstra F, Vellema P, Schneeberger PM, Hackert $V$, ter Schegget R, et al. Sustained intensive transmission of $Q$ fever in the south of the Netherlands, 2009. Euro Surveill. 2009;14(19); pii=19210. Available from: http://www. eurosurveillance.org/ViewArticle.aspx?Articleld=19210

5. van der Hoek W, Dijkstra F, Schimmer B, Schneeberger PM, Vellema P, Wijkmans C, et al. Q fever in the Netherlands: an update on the epidemiology and control measures. Euro Surveill. 2010; 15(12): pii=19520. Available from: http://www. eurosurveillance.org/ViewArticle.aspx?Articleld=19520

6. Roest HI, Tilburg JJ, van der Hoek W, Vellema P, van Zijderveld FG, Klaassen $\mathrm{CH}$, et al. The Q fever epidemic in the Netherlands: history, onset, response and reflection. Epidemiol Infect. 2011;139(1):1-12.

7. Schimmer B, Notermans DW, Harms MG, Reimerink JH, Bakker J, Schneeberger $P$, et al. Low seroprevalence of $Q$ fever in the Netherlands prior to a series of large outbreaks. Epidemiol Infect. 2011;140(1):27-35.

8. van der Hoek W, Meekelenkamp JC, Dijkstra F, Notermans DW, Bom B, Vellema P, et al. Proximity to goat farms and Coxiella burnetii seroprevalence among pregnant women. Emerg Infect Dis. 2011;17(12):2360-3.

9. Hogema BM, Slot E, Molier M, Schneeberger PM, Hermans MH, van Hannen EJ, et al. Coxiella burnetii infection among blood donors during the 2009 Q-fever outbreak in the Netherlands. 
Transfusion 2011. doi: 10.1111/j.1537-2995.2011.03250.x. [Epub ahead of print].

10. European Centre for Disease Prevention and Control (ECDC). Risk assessment on Q fever. Stockholm: ECDC; 2010. Available from: http://ecdc.europa.eu/en/publications/ Publications/1005_TER_Risk_Assessment_Qfever.pdf

11. Wegdam-Blans MC, Kampschreur LM, Nabuurs-Franssen MH, Renders NH, Delsing CE, Bijlmer HA (on behalf of the Dutch consensus group diagnosis of chronic Q fever). Nederlandse consensus chronische Q-koorts [Dutch consensus chronic Q fever]. Tijdschr Infect. 2011;6:71-3. Dutch.

12. Landais C, Fenollar F, Thuny F, Raoult D. From acute $Q$ fever to endocarditis: serological follow-up strategy. Clin Infect Dis. 2007;44(10):1337-40.

13. Limonard GJ, Nabuurs-Franssen MH, Dekhuijzen PN, Groot CA. Prevention of $Q$ fever endocarditis. Lancet Infect Dis. 2011;11(2):82-3.

14. Raoult D, Million M, Thuny F, Carrieri P. Chronic Q fever detection in the Netherlands. Clin Infect Dis. 2011;53(11):1170-1.

15. van der Hoek W, Versteeg B, Meekelenkamp JC, Renders NH, Leenders AC, Weers-Pothoff I, et al. Follow-up of 686 patients with acute $Q$ fever and detection of chronic infection. Clin Infect Dis. 2011;52(12):1431-6.

16. Marmion BP, Ormsbee RA, Kyrkou M, Wright J, Worswick DA, Izzo AA, et al. Vaccine prophylaxis of abattoir-associated $\mathrm{Q}$ fever: eight years' experience in Australian abattoirs. Epidemiol Infect. 1990;104(2):275-87.

17. Gidding HF, Wallace C, Lawrence GL, McIntyre PB. Australia's national $Q$ fever vaccination program. Vaccine 2009;27(14):2037-41.

18. Gefenaite G, Munster JM, van Houdt R, Hak E. Effectiveness of the $Q$ fever vaccine: a meta-analysis. Vaccine. 2011;29(3):395-8.

19. Health Council of the Netherlands. Human vaccination against $Q$ fever: second advisory report. The Hague: Health Council of the Netherlands; 2010. Advisory letter. Publication no. 2010/08E. Available from: http://www.gezondheidsraad.nl/ sites/default/files/201008E.pdf

20. Health Council of the Netherlands. Q fever: risk of transmission via blood or other body material. The Hague: Health Council of the Netherlands; 2011. Advisory letter. Publication no. 2011/15E. Available from: http://www.gezondheidsraad.nl/ sites/default/files/201115EQfever.pdf

21. Morroy G, Peters JB, van Nieuwenhof M, Bor HH, Hautvast JL, van der Hoek W, et al. The health status of $Q$-fever patients after long-term follow-up. BMC Infect Dis. 2011;11:97.

22. Hogerwerf L, van den Brom R, Roest HI, Bouma A, Vellema P, Pieterse $M$, et al. Reduction of Coxiella burnetii prevalence by vaccination of goats and sheep, the Netherlands. Emerg Infect Dis. 2011;17(3):379-86.

23. Reusken C, van der Plaats R, Opsteegh M, de Bruin A, Swart A. Coxiella burnetii ( $Q$ fever) in Rattus norvegicus and Rattus rattus at livestock farms and urban locations in the Netherlands; could Rattus spp. represent reservoirs for (re) introduction? Prev Vet Med. 2011;101(1-2):124-30. 